\title{
DISTRIBUCIÓN Y CONSUMO DE CERÁMICA INCA EN EL PUCARÁ DE TILCARA (QUEBRADA DE HUMAHUACA, ARGENTINA)
}

\author{
CIRCULATION AND CONSUMPTION OF INCA CERAMICS IN \\ THE PUCARÁ OF TILCARA (HUMAHUACA GORGE, ARGENTINA)
}

\author{
Clarisa Otero ${ }^{1}$
}

\begin{abstract}
Se presentan los resultados del análisis de una parte del conjunto cerámico recuperado en el Pucará de Tilcara durante las excavaciones de Ambrosetti y Debenedetti, desarrolladas a inicios del siglo XX. El estudio estilístico de estas piezas, conservadas en las colecciones de dos museos de la Universidad de Buenos Aires, permitió caracterizar la dinámica de los procesos sociopolíticos que llevaron a la intensa circulación de objetos durante la época prehispánica tardía. A su vez, a partir de la contextualización de piezas incaicas, procedentes de distintas provincias del Tawantinsuyu, se determinaron pautas de consumo diferencial. Se plantea que el uso de diversas categorías de bienes estuvo vinculado a la función social y económica que cumplieron distintos grupos de personas en el Pucará.
\end{abstract}

Palabras claves: ocupación inca, colecciones museológicas, distribución cerámica intrasitio, consumo diferencial.

This paper presents the results of an analysis of part of the ceramic assemblage found at the Pucara of Tilcara during Ambrosetti's and Debenedetti's excavations, that took place at the beginning of the twentieth century. The stylistic study of this pottery, found in the collections of two museums at the Universidad de Buenos Aires, allows a characterization the dynamics of the social and politic processes that encouraged the circulation of objects during late prehispanic times. The location of inca pieces that came from different provinces of Tawantinsuyu demonstrate different consumption patterns. The paper concludes that the use of special categories of goods was tied to the social and economic role that different human groups had at the Pucará.

Key words: Inca occupation, museum collections, intrasite ceramic distribution, differential consumption.

La cerámica inca fue uno de los objetos de mayor connotación simbólica presentes en las provincias del Tawantinsuyu. Su distribución, principalmente en los centros de primera jerarquía, sirvió para remarcar la presencia estatal, legitimar el poder, establecer alianzas y reflejar diferencias sociopolíticas (Morris 1995; Williams 2004). Ciertos estilos alfareros regionales, como el Yavi-Chicha o el Pacajes, también cumplieron con estas funciones al ser considerados por los incas como estilos de prestigio. Incluso en determinados casos se promovió su producción y amplia circulación, llegándose a combinar atributos morfológicos, decorativos y tecnológicos propios de estos estilos con los de la alfarería inca cuzqueña (Calderari y Williams 1991; D'Altroy et al. 1994; 2007).

La colección cerámica del Pucará de Tilcara, una de las más notables del Noroeste argentino en relación con su composición y número, presenta una gran variedad de piezas atribuibles a algunos de estos estilos regionales, de vasijas que imitaron a las cuzqueñas y de ejemplares mixtos. De allí que con el propósito de reflexionar sobre las implicancias de la presencia de estos estilos en diferentes contextos del Pucará se analizó gran parte del conjunto recuperado durante las campañas arqueológicas desarrolladas por Ambrosetti (Zaburlín y Otero 2013) y Debenedetti (1930), el que se conserva en los Museos Etnográfico "J. B. Ambrosetti" y Arqueológico "E. Casanova" de Tilcara (ambos de la Facultad de Filosofía y Letras de la Universidad de Buenos Aires).

Además de caracterizar estilísticamente esta muestra se abordó su distribución dentro del Pucará con la intención de visualizar el rol activo que tuvieron los diferentes estilos en las prácticas sociales. Para ello nos circunscribimos a los conjuntos cerámicos presentes en algunas estructuras caracterizadas como casas-taller y en edificios de rasgos arquitectónicos excepcionales y/o con funciones especiales. De esta

1 Instituto Interdisciplinario Tilcara. Facultad de Filosofía y Letras, Universidad de Buenos Aires. Dr. M. Belgrano 445, CP 4624. Tilcara, Jujuy, Argentina, clarisaotero@yahoo.com.ar 
forma se reflexiona por el consumo diferencial y el significado colectivo de determinados tipos de objetos, siendo que la cerámica se constituyó como uno de los principales recursos visuales del Estado para expresar mensajes e imponer nuevas narrativas.

\section{Pucará de Tilcara}

El Pucará de Tilcara se localiza en el área media de la quebrada de Humahuaca, provincia de Jujuy (Figura 1). Con una superficie de 17,5 ha, este sitio se constituye como uno de los poblados más grandes de esta región. Se trata de un conglomerado emplazado en altura sobre la superficie total de un morro de $80 \mathrm{~m}$ de alto, situado en la margen izquierda del río Grande de Jujuy. Su cota máxima alcanza los $2.500 \mathrm{msm}$.

La configuración del espacio del Pucará demuestra una planificación en la traza edilicia durante toda su historia de ocupación, desde el Período de Desarrollos Regionales hasta el Incaico. Las áreas de entierro, junto a los corrales, se encuentran vinculadas
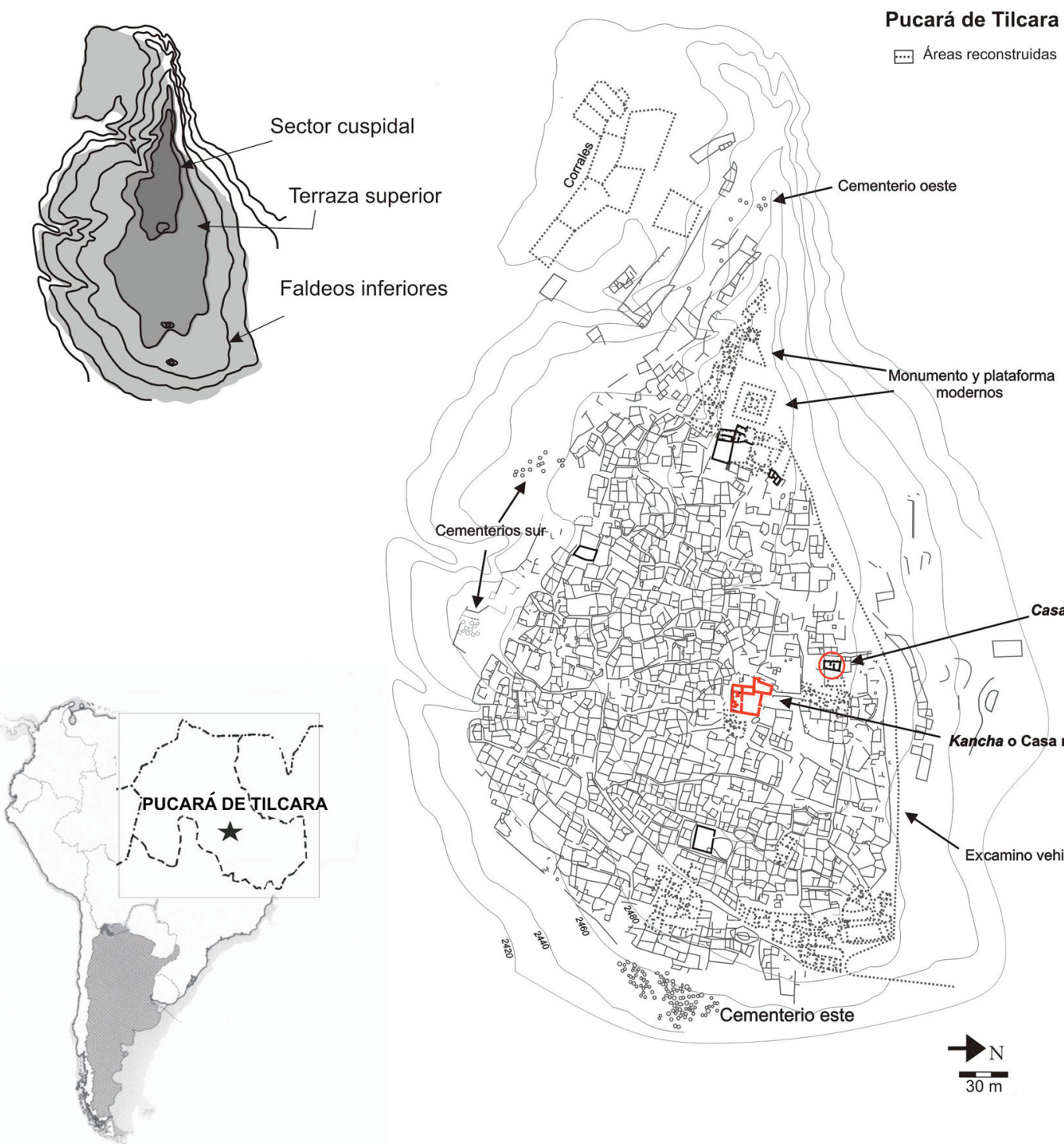

... Áreas reconstruidas

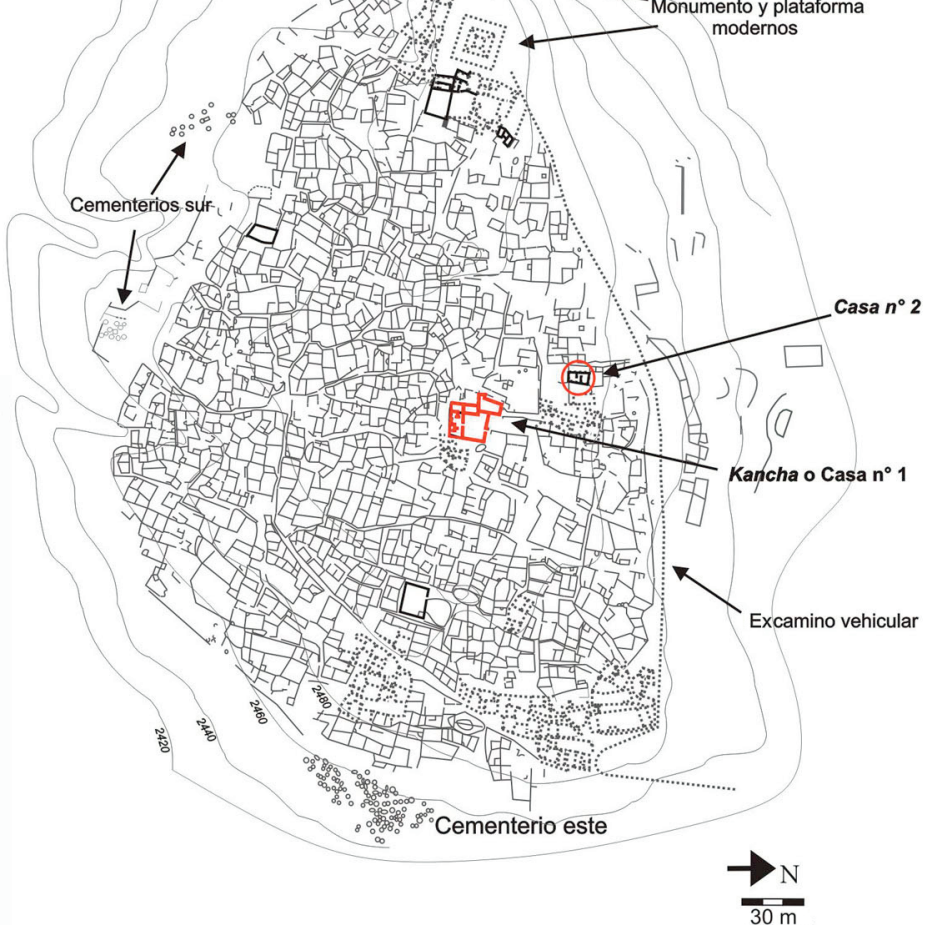

Figura 1. Plano del Pucará de Tilcara. Relevamiento completo realizado por Lanzelotti et al. (2012) sobre el plano original de Zaburlín (2009). Referencias espaciales propuestas por Zaburlín (2009).

Layout of the Pucará of Tilcara, made by Lanzelotti et al. (2012). Space references presented by Zaburlín (2009). 
a las principales vías de acceso y segregadas de los espacios habitacionales y de trabajo artesanal (Figura 1). Entre estos espacios, compuestos por más de 580 estructuras de diversas formas y tamaños dentro de un patrón general cuadrangular, se han identificado plazas y una kancha (Hyslop 1992), la que fue utilizada como edificio ceremonial, tal como se menciona más adelante.

En la actualidad se cuenta con 25 fechados radiocarbónicos (AMS y C14) obtenidos para distintos sectores del sitio. Si bien sus resultados demuestran una larga ocupación del poblado, abarcando un rango temporal que se inicia en el segundo milenio y finaliza durante el siglo XVI d.C., las investigaciones que venimos desarrollando en los últimos años demuestran que las evidencias materiales asociadas a los fechados que refieren a una ocupación previa al siglo XII son escasas (Otero y Rivolta 2013). Asimismo se ha podido establecer que el momento de su máxima ocupación se produjo durante la época incaica (Otero 2013), intervalo en el que el Pucará posiblemente se constituyó como capital de la Wamani o provincia de Humahuaca (González 1982; Williams 2004).

Además de funcionar como un enclave político y administrativo de primera jerarquía, este sitio fue uno de los principales centros productivos de la región. En él se instalaron numerosos talleres artesanales destinados a la manufactura de bienes ornamentales y de uso simbólico. Entre ellos se pueden mencionar vasos, discos, tumis y tupus elaborados en diversos metales, como oro, plata, bronce y cobre. Por otro lado, confeccionados en distintas variedades de valvas y rocas, entre ellas ónix, caliza, sílice y alabastro, se produjeron pendientes, placas, recipientes y tallas posiblemente utilizadas como illas (Krapovickas 1958/1959; Otero 2013). Estas materias primas también se aprovecharon para confeccionar torteros de roca y cuchillos, cinceles y punzones de metal.

A partir de nuestros trabajos de excavación, la información vertida en las publicaciones y manuscritos de Ambrosetti (Zaburlín y Otero 2013) y de Debenedetti (1930), y de la recomposición de los contextos de aparición de 659 objetos confeccionados en distintas materialidades, depositados en los museos mencionados, se han podido contabilizar más de cincuenta talleres distribuidos a lo largo de todo el Pucará (Otero 2013). Estos talleres en algunos casos estuvieron destinados a ambas producciones, tanto de objetos de piedra como de metal.
La cantidad y el tipo de evidencias que reflejan el desarrollo de estas producciones han servido para definir el grado de especialización de las mismas, particularmente de la industria lapidaria. A partir de nuestras investigaciones contabilizamos treinta y dos talleres dedicados al trabajo en piedra, ubicados en los sectores más elevados del Pucará. Estos talleres se caracterizan por presentar un importante número de preformas de los objetos que se confeccionaban en roca y no de sus formas acabadas. Se trata de más de un centenar de preformas de torteros de caliza de iguales dimensiones y de tallas incompletas de pendientes trabajados en alabastro, similares a los hallados por Bingham en Machu Pichu (Rowe 1946: 316, lám. 79a). Estas evidencias sugieren que parte de esta producción se desarrolló a gran escala y de forma estandarizada. Asimismo, la escasez de formas completas podría indicar que estos bienes fueron trasladados para ser utilizados por fuera del Pucará. Entre los numerosos materiales de la colección procedente de este sitio solo se han detectado formatizados de manera acabada tres illas, un pendiente de alabastro y dos torteros de caliza. Por otro lado, hasta el momento no se han identificado evidencias de su aprovechamiento a nivel regional. De allí que se considere que estos bienes, una vez finalizada su manufactura, fueron transportados a otras provincias del Tawantinsuyu (Otero 2013).

Si bien en los Andes del Sur los recursos mineros metalíferos fueron los más valorados por los incas para su explotación (Williams et al. 2009), en el caso de Tilcara, la presencia de fuentes de distintas variedades de roca para el desarrollo de la industria lapidaria debió resultar una gran ventaja. La gran cantidad y diversidad de tallas y recipientes de alabastro presentados por Valcárcel (1934a, 1934b, 1934c, 1935a, 1935b), hallados como inclusiones mortuorias de numerosas sepulturas excavadas en Sacsahuaman, demuestran la afinidad que los incas tenían por este tipo de artesanías. La posibilidad de producir estos objetos en el Pucará, sumado al control de la ruta hacia el sur que proveía este sitio, por encontrarse ubicado en el sector central del corredor natural en que se constituye la quebrada de Humahuaca, debió posicionarlo como un enclave regional de una importancia fundamental para el Estado. De allí que con la intención de dar cuenta desde otra línea de evidencia de la relevancia que tuvieron en la economía incaica la producción de estas manufacturas junto a la de diversos objetos de metal, 
en este trabajo se busque caracterizar a los conjuntos cerámicos recuperados en algunos de los edificios que fueron habitados por personas vinculadas a la política de producción estatal. A continuación se presentan las características estilísticas del total de la muestra cerámica analizada, para luego abordar la distribución de ciertas categorías de objetos.

\section{Características de la Muestra Cerámica}

El conjunto analizado se compone por 305 piezas cerámicas que fueron agrupadas en 16 clases formales. Estas clases se definieron según los criterios morfológicos utilizados por diferentes autores que han trabajado con muestras de la quebrada de Humahuaca (Cremonte 2006; Deambrosis y De Lorenzi 1973; López 2004; Nielsen 2001; Otero 2006, 2013; Otero y Cremonte 2014; Palma 1998; Runcio 2009), y para el caso de la cerámica inca, a partir de las categorías propuestas por investigadores que estudiaron conjuntos de los Andes Centrales y de distintas provincias del Tawantinsuyu (Pardo 1939; Rowe 1944; Meyers 1975; Julien 2004; Bray 2004). Esta diversidad de formas abarca tanto a estilos locales como no locales (Tablas 1 y 2).

Si bien cada clase formal presenta variedades internas según el tamaño, contorno general de la vasija o tipo de apéndices, la mayoría de las piezas corresponden a formas más bien pequeñas. A excepción de ocho cántaros y tres ollas, cuyas alturas superan los $50 \mathrm{~cm}$, el resto de las vasijas no alcanzan $\operatorname{los} 30 \mathrm{~cm}$ de altura. Esto responde principalmente al sesgo que se identifica al estudiar colecciones, ya que con frecuencia las grandes ollas y cántaros exhumados durante las primeras excavaciones no pasaban a formar parte de las colecciones de los museos (Palma 1998).

El $82 \%$ de la muestra está representado por los estilos cerámicos tardíos de Humahuaca y por el conjunto de piezas Inca Provincial local o Humahuaca Inca (sensu Cremonte 2006; Nielsen 2001) (Tabla 1). Dentro de este grupo predominan los pucos y cántaros Humahuaca N/R, los pucos Poma N/R y los Humahuaca Inca N/R.

La cerámica no local corresponde al $18 \%$ de la muestra analizada. Este grupo incluye piezas que proceden de regiones trasandinas, altiplánicas y selváticas. Entre las 55 vasijas que lo componen, 23 son del tipo Inca Provincial. Por un lado, siete corresponden a estilos regionales incaicos como el Inca Pacajes ${ }^{1}$, Inca Paya e Inca Yavi-Chicha (Cremonte
2014) (Tabla 2). Estas piezas posiblemente circularon por los Andes del Sur por canales paralelos a los de la distribución de la cerámica Inca Polícroma (Williams 2004). Por otro lado, dentro de este conjunto se identificaron nueve vasijas que si bien en algunos casos se han registrado ejemplares similares en otros sitios del Noroeste argentino por el momento no se puede establecer su lugar de origen, de allí que se las denominara Inca Provincial Indeterminado. Se trata de una olla morada con pie (MEJBA 35143- MT $2285)^{2}$, cinco platos ornitomorfos, dos de ellos negros bruñidos (MEJBA 3757- MT 2216 y MEJBA 3482), una jarra marrón pulida del tipo Puchuela (Palma 1998:29; Pardo 1939:11) (MEJBA 3618-MT 2575), un aríbalo morado pulido decorado en el cuello por una franja negra (MEJBA 8612), y un plato rojo pulido de asa de doble inserción (sensu Deambrosis y de Lorenzi 1973:132). Este último presenta en su base interna una cruz negra conformada por líneas finas que rematan en forma de peine (Pardo 1939: 19) (MEJBA 35103) (Figura 2).

Entre las piezas incaicas solo se detectó una de origen cuzqueño. Se trata de un aríbalo Cuzco Polícromo Figurativo (sensu Rowe 1944: 48) (MEJBA 7604) (Tabla 2) (Figura 3). Otro conjunto de piezas excepcionales son siete vasijas que imitan a las Imperial (Tabla 2). Este conjunto no condice con las producciones cerámicas del centro del Imperio. Carecen de algunos elementos morfodecorativos propios de la vajilla imperial o su manufactura es burda. Un ejemplo de ello es una jarra tipo Aysana (Palma 1998:29; Pardo 1939:10), decorada de forma desprolija con diez bandas verticales de líneas rojas rellenas por tres conjuntos de triángulos marrones unidos por el vértice a manera de reloj de arena (MEJBA 8610) (Figura 3). Por otro lado, en un plato fragmentado de asa de doble inserción (sensu Deambrosis y De Lorenzi 1973:132) (MEJBA 8734), de características similares a las de un ejemplar hallado en Machu Pichu por Bingham (1913:212), el trazo de las líneas quebradas que componen la banda central del interior de la pieza también es irregular. Por último, los cinco aríbalos polícromos identificados para este grupo presentan una estructuración del diseño y elementos típicos de la alfarería cuzqueña, como el motivo de helecho o las hileras de rombos (Rowe 1944:47) (MEJBA 8732, 8733, 35100, 3787 y MEJBA 20864-MT 2546). No obstante se encuentran decorados de forma descuidada, a tal punto que se rompe con la composición simétrica del diseño. 


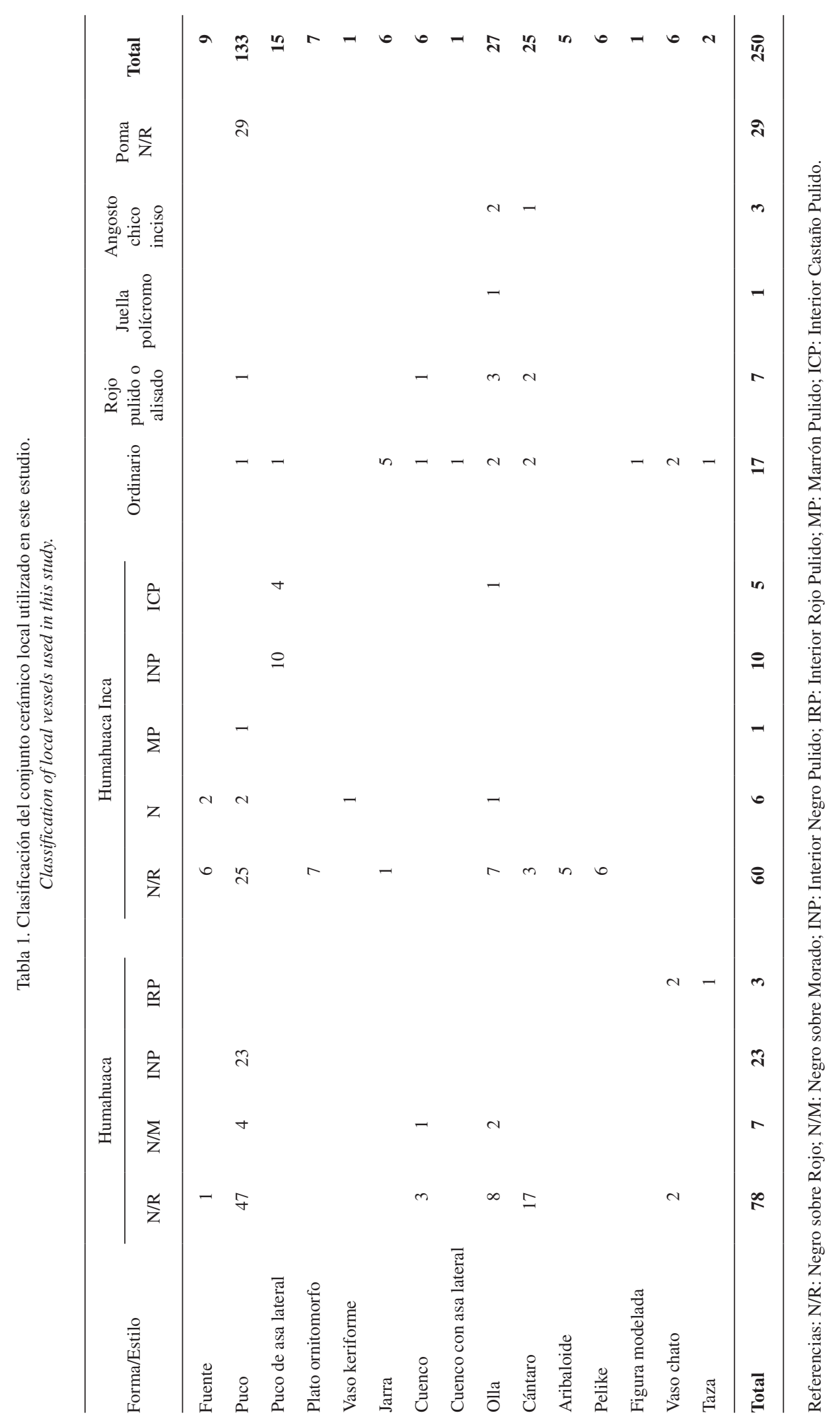




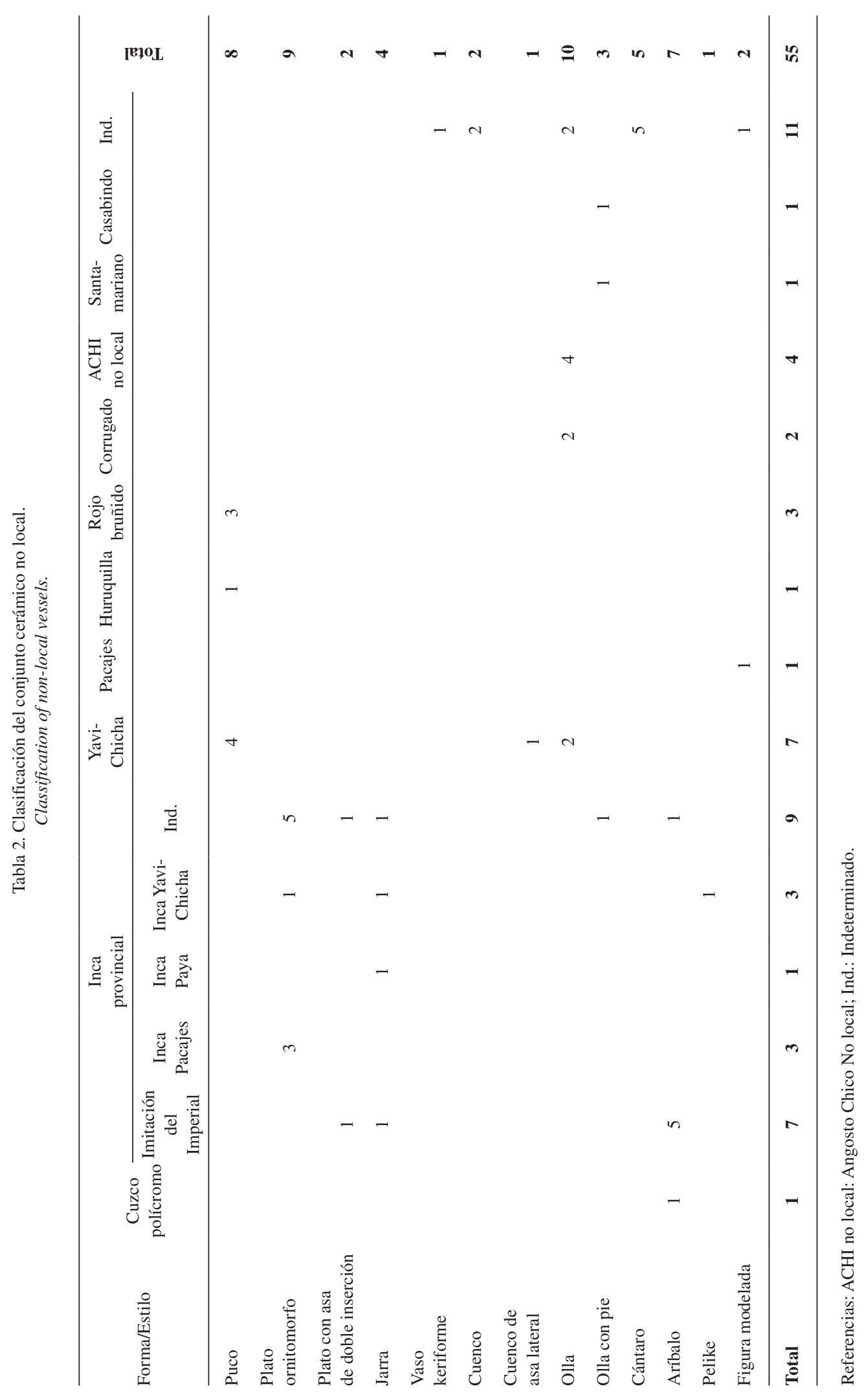




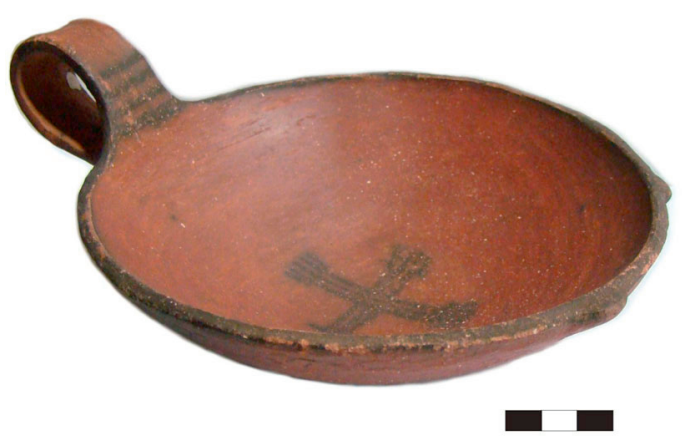

Figura 2. Plato Inca Provincial (MEJBA 35103). Provincial Inca style plate (MEJBA 35103).

En su totalidad, la importante representación de piezas incaicas y de vasijas correspondientes a estilos regionales no incaicos de alta calidad tecnológica, como los pucos Rojo Bruñido, similares a los descritos por Cremonte y Botto (2009), o las piezas Yavi-Chicha (Cremonte 2014) (Tabla 2), posiblemente responda a que a medida que avanzó la anexión de territorios hacia el sur, la quebrada debió funcionar como uno de los principales corredores de articulación política y económica de la región. Sus vías de comunicación, tanto con las tierras altas como con las yungas, debieron llevar a la circulación de personas y objetos que se incorporaron en la esfera social de los habitantes del Pucará. Asimismo, esta representación es coherente con las funciones que debió cumplir este sitio como posible centro provincial, contemplando que estos tipos de cerámica fueron considerados un bien de prestigio, en ocasiones utilizados para representar al poder estatal (Williams 2004). Para determinar en qué prácticas fueron empleadas y quiénes tuvieron acceso a estas piezas se buscó reconstruir las características del contexto de aparición de cada una de ellas.

Por un lado se trabajó con los catálogos de ingreso de los materiales de ambos museos con el propósito de establecer la procedencia de los objetos. Esta información fue ampliada con la de los diarios de campo y las publicaciones de Ambrosetti (Zaburlín y Otero 2013) y de Debenedetti (1930). El carácter descriptivo de estos trabajos sirvió para reconstruir las características de los espacios abordados y de los materiales recuperados. Si bien se reconoce que muchas de las estructuras y entierros de donde proceden las piezas pudieron corresponder a sucesivas ocupaciones del sitio, fue mediante estas descripciones que se logró delimitar espacial y temporalmente a los conjuntos. Durante el estudio de las colecciones se priorizó la búsqueda de ciertos materiales con el propósito de recomponer aquellos contextos que a partir de las descripciones de Ambrosetti y Debenedetti o las vertidas en los catálogos de los museos se daba cuenta de su valor para la caracterización de determinados conjuntos de bienes y de las actividades desarrolladas en cada espacio.

Entre las 305 piezas analizadas, 64 no presentan información sobre su ubicación dentro del Pucará. Las 241 restantes se distribuyeron entre 96 construcciones diferentes localizadas en el sector cuspidal, la terraza

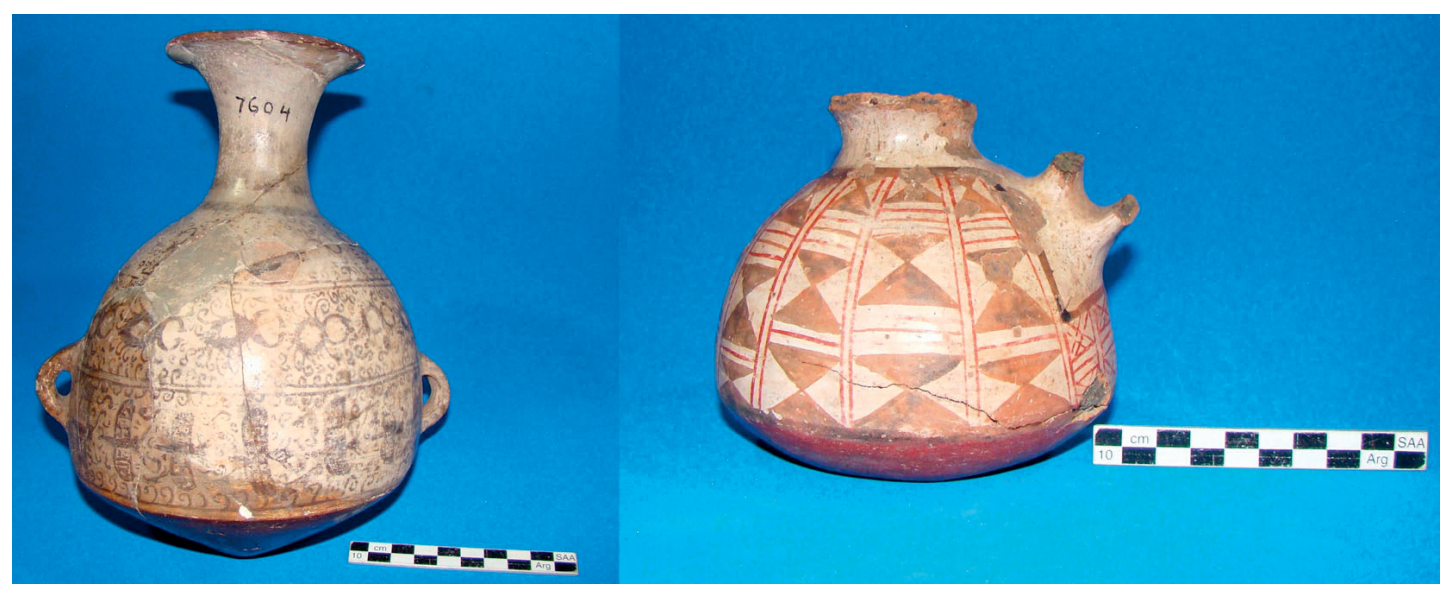

Figura 3. Aríbalo Cuzco Polícromo Figurativo (MEJBA 7604) y jarra polícroma (MEJBA 8610).

Figurative Polychrome Cuzco aryballos (MEJBA 7604) and polychrome jar (MEJBA 8610). 
superior y la porción alta de los faldeos sur, sureste y suroeste (Figura 1). De estas 96 estructuras, en este trabajo se analizan los contextos de solo 16 edificios. Principalmente se debe a que se trata de espacios donde se pudieron establecer asociaciones claras entre materiales, y para ellos se ha podido determinar con precisión su función.

\section{La Cerámica de los Talleres y Edificios Especiales}

Para avanzar sobre la existencia de pautas de consumo diferencial de la cerámica, tal como se mencionó, se analizaron 16 estructuras del Pucará. Doce de ellas corresponden a talleres destinados a la producción lapidaria y metalúrgica. Los cuatro restantes son edificios de características especiales. Tal como se presenta más adelante, en estos últimos se hallaron algunas de las piezas que imitan a las Imperiales y el aríbalo Cuzco Polícromo Figurativo anteriormente descrito.

En relación con los talleres, se cuenta con la ubicación precisa de cinco de ellos, los que fueron excavados durante las primeras intervenciones al Pucará dirigidas por Ambrosetti (Zaburlín y Otero 2013). Se trata de las estructuras por él denominadas como Casa de los Pedernales, Casa de Altamirano, Casa de los Torteros, Casa de los Platos Pato y la Casa $\mathrm{n}^{\circ} 2$, que a diferencia de las anteriores fue descrita por Debenedetti (1930:45). Las primeras cuatro Casas se encontraban donde se edificó en 1935 el monumento realizado por Casanova en homenaje a sus maestros (Figura 1). A partir de las referencias presentadas en el manuscrito de Ambrosetti, Zaburlín pudo identificar la ubicación de algunas de ellas (Zaburlín y Otero 2013). Por otro lado, la Casa ${ }^{\circ} 2$ se encuentra en la terraza superior, en las proximidades del faldeo Norte (Figura 1). Los talleres restantes, enumerados en los catálogos del Museo Etnográfico como Casas n ${ }^{\circ} 3,10,34$, $35,36,62$ y 69, posiblemente se localizaban en la terraza superior y el faldeo sur (Figura 1).

Estos talleres pueden definirse como casas-taller debido a que en ellos se han detectado evidencias que hacen referencia tanto a actividades domésticas como de producción multiartesanal (Shimada 2007). Las evidencias que refieren a estas últimas actividades son: escorias, minerales, herramientas -como martillos, pulidores, punzones y astas de taruca-, tapones de cuchara, moldes y refractarios, utilizados para el fundido y moldeado de objetos de metal, y producciones incompletas, como preformas de torteros de caliza y de tallas confeccionadas en ónix, sílice y alabastro (Tabla 3).

La cerámica de estos contextos es atribuible a los estilos tardíos de la quebrada, algunos de estos continuaron en uso durante la ocupación incaica, y principalmente a variedades Humahuaca Inca, como cántaros, aribaloides y pucos $\mathrm{N} / \mathrm{R}$, y pucos de asa lateral Interior Negro Pulido. Un aspecto notorio es que en varios de estos contextos se han identificado piezas foráneas. A manera de ejemplo, en la Casa de los Torteros y en la de los Platos Pato se detectaron numerosos platos ornitomorfos, entre ellos algunos ejemplares estilo Inca Yavi-Chicha (MEJBA 3373) (Figura 4); en las Casas nos 2 y 36 se recuperaron platos ornitomorfos Inca Pacajes (MEJBA 3716MT 2474 y MEJBA 7438); en las Casas ${ }^{\text {os }} 35$ y 69 se identificaron pucos Rojo Bruñido (MEJBA 7505-MT 2586 y MEJBA 4667); en esta última Casa también se registró un puco Yavi-Chica (MEJBA 4367-MT 2367), y en la Casa ${ }^{\circ} 34$ se halló una olla de tipo Corrugado (MEJBA 8137), que como se determinó constituye un estilo cuyo origen se registra en el borde oriental y meridional de la quebrada, apareciendo en la región para momentos incaicos (Cremonte 2006; Nielsen 2001) (Tabla 3).

Los cuatro edificios donde fueron detectadas las piezas que imitan a las cuzqueñas y el aríbalo Cuzco Polícromo Figurativo son la kancha, que en los catálogos del Museo Etnográfico figura como Casa $n^{\circ} 1$, ubicada en el sector central del Pucará (Figura 1); la Casa $n^{\circ} 55$, posiblemente edificada entre este centro ceremonial y el sector cuspidal; el Yacimiento ${ }^{\circ}{ }^{3}$, excavado por Debenedetti en 1929, aparentemente localizado en el tramo superior del faldeo sureste, y por último, el Yacimiento $\mathrm{n}^{\circ}$ 98, para este por el momento no se ha podido determinar su ubicación.

La kancha es localmente conocida como "La Iglesia". Este edificio ceremonial, de más de 220 $\mathrm{m}^{2}$, se compone por un conjunto de recintos de diferentes formas y tamaños. En el interior de uno de sus recintos, denominado Edificio A, se detectó una serie de rasgos y construcciones que en su conjunto resultan únicos en la arquitectura prehispánica de la quebrada. Además de un nicho en una de sus paredes, se identificó una estructura rectangular de no más de un metro de altura, construida con rocas canteadas (Figura 5). Debenedetti (1930:40) la describe como "una especie de altar", que al iniciar las excavaciones en 1908 se encontraba expuesto. 
Tabla 3. Síntesis de los conjuntos de hallazgos recuperados en las casas-taller analizadas. Brief description of the material assemblages recovered from the analyzed household workshops.

\begin{tabular}{|c|c|c|}
\hline $\begin{array}{l}\text { Nombre o } \\
\mathrm{N}^{\circ} \text { de la } \\
\text { casa-taller }\end{array}$ & $\begin{array}{l}\text { Hallazgos vinculados al desarrollo de } \\
\text { actividades productivas especializadas }\end{array}$ & $\begin{array}{l}\text { Registro de algunas de las piezas cerámicas } \\
\text { identificadas }\end{array}$ \\
\hline $\begin{array}{l}\text { Casa de los } \\
\text { Pedernales }\end{array}$ & $\begin{array}{l}\text {-Perforadores líticos } \\
\text {-Alisadores líticos } \\
\text {-Pulidores líticos } \\
\text {-Lascas y núcleos de sílex y obsidiana. }\end{array}$ & $\begin{array}{l}\text {-Cántaro antropomorfo Humahuaca N/R } \\
\text {-Puco de asa lateral Humahuaca Inca INP } \\
\text {-Dos ollas ACHI de manufactura no local } \\
\text {-Olla Humahuaca Inca N/R } \\
\text {-Dos ollas Humahuaca Rojo Pulido }\end{array}$ \\
\hline $\begin{array}{l}\text { Casa de } \\
\text { Altamirano }\end{array}$ & $\begin{array}{l}\text {-Preformas de torteros de caliza } \\
\text {-Fragmentos de rocas marmóreas } \\
\text {-Trozos de obsidiana }\end{array}$ & $\begin{array}{l}\text {-Puco Humahuaca Inca Marrón Pulido } \\
\text {-Puco Humahuaca Inca N/R } \\
\text {-Puco Poma N/R }\end{array}$ \\
\hline $\begin{array}{l}\text { Casa de los } \\
\text { Platos Pato }\end{array}$ & $\begin{array}{l}\text {-Preformas de torteros de caliza } \\
\text {-Preformas de pendientes de alabastro } \\
\text {-Núcleos y lascas de rocas marmóreas } \\
\text {-Cuarzos trabajados } \\
\text {-Discos de piedra laja } \\
\text {-Fragmentos de valvas de moluscos }\end{array}$ & $\begin{array}{l}\text {-Dos platos ornitomorfos Inca Yavi-Chicha } \\
\text {-Fragmentos de piezas Humahuaca Inca N/R }\end{array}$ \\
\hline $\begin{array}{l}\text { Casa de los } \\
\text { Torteros }\end{array}$ & $\begin{array}{l}\text {-Discos de piedra laja } \\
\text {-Trozos de mármol } \\
\text {-Preformas de torteros de caliza } \\
\text {-Afilador de romanechita } \\
\text {-Fragmentos de alabastro y sílex } \\
\text {-Manos/pulidores de piedra } \\
\text {-Espátulas de hueso } \\
\text {-Astas de taruca }\end{array}$ & $\begin{array}{l}\text {-Puco Humahuaca Inca N/R } \\
\text {-Platos ornitomorfos Humahuaca Inca N/R } \\
\text {-Dos platos ornitomorfos Inca Yavi-Chicha }\end{array}$ \\
\hline 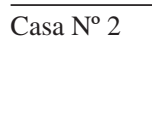 & $\begin{array}{l}\text {-Trozos de mineral de cobre } \\
\text {-Espátula de hueso } \\
\text {-Punzón de metal } \\
\text {-Fragmentos de objetos de metal (tumis, tupus) }\end{array}$ & $\begin{array}{l}\text {-Plato ornitomorfo Inca Pacajes } \\
\text {-Aribaloide Humahuaca Inca N/R } \\
\text {-Olla con pie Inca Provincial Indeterminado } \\
\text {-Olla Humahuaca Rojo Pulido }\end{array}$ \\
\hline Casa 3 & $\begin{array}{l}\text {-Trozos de mineral de cobre } \\
\text {-Mortero y mano de roca, ambos con } \\
\text { impregnaciones de mineral de cobre. }\end{array}$ & $\begin{array}{l}\text {-Puco Poma N/R } \\
\text {-Puco de asa lateral Humahuaca Inca INP } \\
\text {-Cántaro Humahuaca Inca N/R } \\
\text {-Pieza subesferoidal con pico Morado Pulido, de origen no local }\end{array}$ \\
\hline Casa 10 & $\begin{array}{l}\text {-Yeso } \\
\text {-Fragmentos de objetos de metal } \\
\text {-Martillo lítico } \\
\text {-Pulidor lítico }\end{array}$ & $\begin{array}{l}\text {-Puco de asa lateral Humahuaca Inca INP } \\
\text {-Puco Humahuaca Inca N/R } \\
\text {-Puco Humahuaca Inca Marrón Pulido }\end{array}$ \\
\hline Casa 34 & $\begin{array}{l}\text {-Instrumentos de hueso } \\
\text {-Alisador de hueso } \\
\text {-Astas de taruca } \\
\text {-Fragmentos de ocre rojo } \\
\text {-Fragmentos de valvas de molusco } \\
\text {-Cinceles de bronce }\end{array}$ & $\begin{array}{l}\text {-Olla de estilo Corrugado } \\
\text {-Cántaro Humahuaca Inca N/R } \\
\text {-Puco de asa lateral Humahuaca Inca INP }\end{array}$ \\
\hline Casa 35 & $\begin{array}{l}\text {-Cincel de metal } \\
\text {-Mortero de piedra } \\
\text {-Tapón de cuchara de metalurgia }\end{array}$ & $\begin{array}{l}\text {-Puco Rojo Bruñido } \\
\text {-Aribaloide Humahuaca Inca N/R } \\
\text {-Tres pucos Poma N/R } \\
\text {-Olla ACHI de manufactura no local } \\
\text {-Pucos de asas lateral Humahuaca Inca INP }\end{array}$ \\
\hline Casa 36 & $\begin{array}{l}\text {-Fragmentos de valvas } \\
\text {-Discos de cuarzo perforados } \\
\text {-Escorias } \\
\text {-Trozos de mineral de cobre y arcilla } \\
\text {-Preformas de torteros de roca }\end{array}$ & $\begin{array}{l}\text {-Pucos Poma N/R } \\
\text {-Olla con pie Inca Provincial Indeterminado } \\
\text {-Plato ornitomorfo Inca Pacajes } \\
\text {-Olla Humahuaca Inca N/R } \\
\text {-Olla Humahuaca N/R }\end{array}$ \\
\hline Casa 62 & $\begin{array}{l}\text {-Trozos de mineral de cobre } \\
\text {-Preformas de torteros de roca }\end{array}$ & $\begin{array}{l}\text {-Olla Humahuaca N/M } \\
\text {-Olla Humahuaca Inca N/R } \\
\text {-Pucos Humahuaca Inca N/R y CP }\end{array}$ \\
\hline Casa 69 & $\begin{array}{l}\text {-Tapón de cuchara de metalurgia } \\
\text {-Asta de taruca } \\
\text {-Martillo lítico }\end{array}$ & $\begin{array}{l}\text {-Seis pucos Humahuaca N/R } \\
\text {-Puco Poma N/R } \\
\text {-Dos pucos Interior Negro Pulido } \\
\text {-Puco Rojo Bruñido } \\
\text {-Puco Yavi-Chicha }\end{array}$ \\
\hline
\end{tabular}




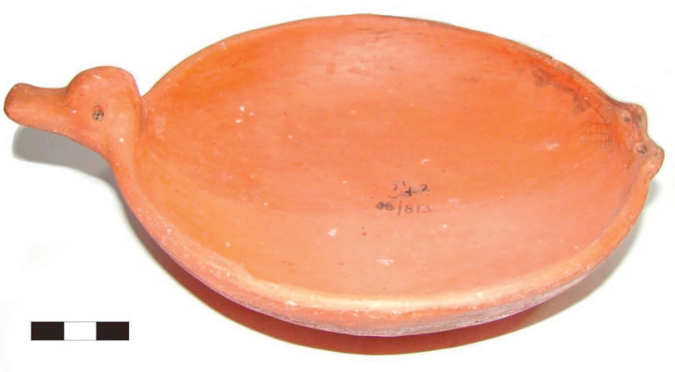

Figura 4. Plato Inca Yavi-Chicha (MEJBA 3373). Inca Yavi-Chicha plate (MEJBA 3373).

Junto a este "altar" se halló un vaso modelado en arcilla. Se trata de la figura de un sapo de $13 \mathrm{~cm}$ de largo estilo Pacajes (MEJBA 3707-MT 2242) (Figura 5). Esta figura presenta una pequeña perforación en su boca, posiblemente utilizada para libar chicha. Asociados a este vaso se recuperaron el cuello de un cántaro Humahuaca N/R (MEJBA 3391) y dos piezas rojas pulidas, según Debenedetti (1930: 41) de fina manufactura. Por el momento no se realizó el análisis estilístico de estas dos últimas vasijas, ya que no pudieron ser localizadas dentro de la colección del Museo Etnográfico.

En una de las dos estructuras cuadrangulares que se ubican sobre el muro sur de este edificio, Debenedetti identificó dos platos ornitomorfos, uno de ellos decorado con el motivo que Rowe (1944:47) describiera como banda central compuesta por el patrón de "X" combinado con líneas paralelas verticales (MEJBA 3708-MT 2284) (Figura 5). Para la segunda estructura, Debenedetti menciona que en ella solo se recuperó un aríbalo, el que tampoco se ha podido localizar dentro de la colección del Museo Etnográfico. Asimismo, para distintos sectores de este edificio, este autor señala el hallazgo de numerosos fragmentos de "vasos finos" (Debenedetti 1930: 41 ), como ollas con pie y piezas restringidas con decoración reticulada en negro sobre fondo blanco (MEJBA 3787).

Otros objetos también demuestran el carácter ceremonial de esta estructura. Entre ellos se han detectado una de las tres únicas illas de alabastro que fueron recuperadas de forma completa en el Pucará, una valva de Pecten sp., una lentejuela de cobre, posiblemente utilizada como adorno, un fragmento de bastón de madera, una espátula para inhalar alucinógenos, una aguja de hueso quizás empleada para limpiar los tubos de inhalación de estas sustancias, una figurilla zoomorfa confeccionada en lutita y un fragmento de nuez formatizado a manera de cascabel. Al reabrir las excavaciones en este edificio luego del fallecimiento de Debenedetti, Casanova encuentra entre las dos estructuras de la pared sur un cráneo sepultado (Casanova 1970:37). Este hallazgo se suma a las inhumaciones descubiertas por Debenedetti de dos individuos ubicados en la esquina sureste de este recinto (Debenedetti 1930:41).

El conjunto de evidencias halladas en este edificio da cuenta del desarrollo de variadas prácticas rituales que, además de la promulgación de las bases religiosas incaicas, habrían tenido como propósito lograr la organización de la producción en función de los intereses del Estado (Otero 2013; Otero y Ochoa 2011). En este marco escénico, entre la vajilla incaica, particularmente los aríbalos debieron utilizarse para el desarrollo de los rituales de consumo de chicha (Cook y Glowacki 2003) con la intención de cumplir con la prestación estatal de esta bebida que, tal como indica Bray (2004), fue más importante en las regiones distantes del Cuzco que en el propio centro del Imperio. Por otro lado, los platos ornitomorfos y las ollas con pie pudieron ser parte de la cerámica utilizada por los funcionarios y militares que quizás habitaron algunos de los recintos de esta kancha.

Junto a los aríbalos, los platos y las ollas con pie constituían la tríada por excelencia de los equipos de cocina de estas personalidades en las provincias (Bray 2004). Vale mencionar que este tipo de piezas también se identificó en los conjuntos de hallazgos de los edificios restantes. En la Casa $\mathrm{n}^{\circ} 55$ del catálogo del Museo Etnográfico, que corresponde al Yacimiento 167 de la publicación de Debenedetti (1930:101), se identificaron tres cámaras mortuorias, una de ellas se encontró vacía. En otra de ellas se detectó el entierro de un individuo adulto, que incluidos como ofrendas poseía una pala de madera y dos platos ornitomorfos, según menciona Debenedetti del tipo cuzqueño. En la tercera se recuperaron dos esqueletos de adultos junto a otros dos platos ornitomorfos, una pala de madera, una gran fuente Humahuaca Inca N/R, tres ollitas rojas, una con decoración geométrica, un puco negro, el aríbalo Cuzco Polícromo Figurativo (MEJBA 7604) y la jarra polícroma (MEJBA 8610) anteriormente descritos (Figura 3). Durante la revisión de las colecciones, entre las numerosas piezas analizadas para este edificio, además se identificaron una jarra Inca Paya (MEJBA 8611), 
una ollita de estilo Corrugado (MEJBA 7142-MT 2241) y un cantarito con pico de presunto origen boliviano (MEJBA 8614).

Por otro lado, en un entierro del Yacimiento $\mathrm{n}^{\circ}$ 98 se recuperaron un cántaro Humahuaca Inca N/R, dos platos ornitomorfos, y dos fragmentos de cuello y borde de uno de los aríbalos polícromos mencionados, que dentro del conjunto Inca Provincial imitan a las piezas cuzqueñas (MEJBA 8732). Por último, en una esquina del Yacimiento $n^{\circ} 35$ se recuperó el plato con asa de doble inserción (MEJBA 35103) (Figura 2) y el aríbalo Inca Provincial (MEJBA 35100) (Figura 6). Asimismo se encontraron dos aribaloides Humahuaca Inca N/R, uno de estos presenta bubones (MEJBA 35102-MT 2196 y MEJBA 35101-MT 2252).

A excepción de una mano de moler y un mortero hallados en este último Yacimiento, no se detectaron otros elementos que evidencien el desarrollo de actividades productivas in situ. De igual forma sucede con la Casa ${ }^{\circ} 55$ y el Yacimiento $n^{\circ} 98$. De allí que si se considera la ausencia de este tipo de elementos, sumada a la presencia de alfarería que imita a la Imperial y de ciertos rasgos arquitectónicos que no resultan frecuentes en la gran mayoría de las estructuras habitacionales del Pucará (hornacinas, pisos enlajados o la utilización de rocas canteadas), estas Casas y Yacimientos, incluida la kancha, podrían ser considerados como las residencias de los representantes del Estado. Entre estos representantes se debió contar con personas de distintas jerarquías involucradas tanto en el sistema político-administrativo como religioso, e incluso, como se ha tratado para otros casos, quizás se haya incluido a miembros de la elite local (González y Tarragó 2004; Acuto 2010; Santoro et al. 2010).

A lo largo del Pucará existieron otras viviendas con estas características, las que han sido identificadas a partir de las descripciones mencionadas en los catálogos y en las libretas de campo de Debenedetti (1930). La distribución de estas estructuras demuestra que se ubicaban entre los talleres de los diferentes sectores del Pucará, posiblemente con el propósito

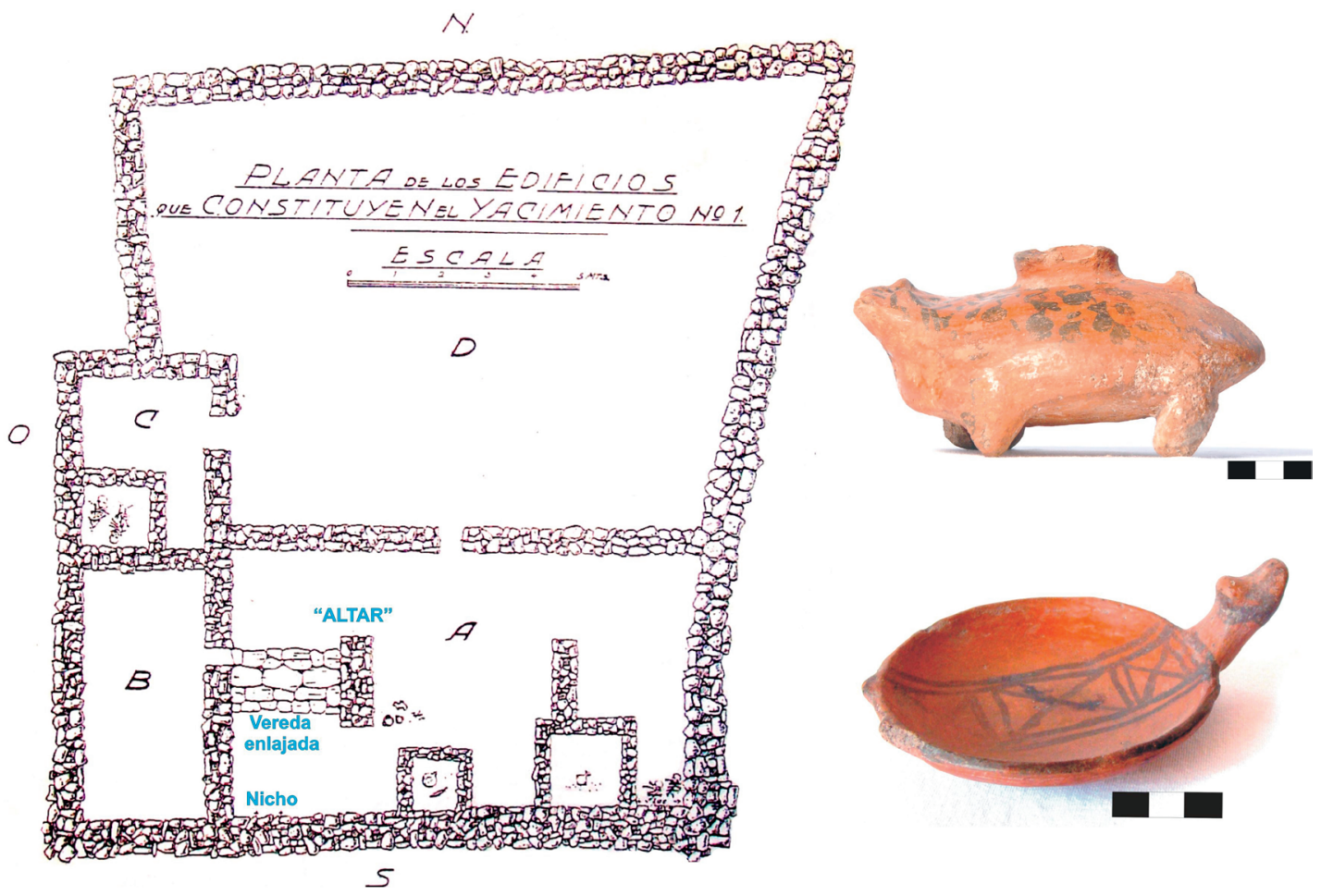

Figura 5. Planta de "La Iglesia", modificada de Debenedetti (1930:39). Figura zoomorfa Pacajes (MEJBA 3707-MT 2242) y plato Inca Provincial (MEJBA 3708-MT 2284).

Layout of "The Church", modified from Debenedetti (1930:39). Pacajes zoomorphic figure (MEJBA 3707-MT 2242) and Provincial Inca style plate (MEJBA 3708-MT 2284). 


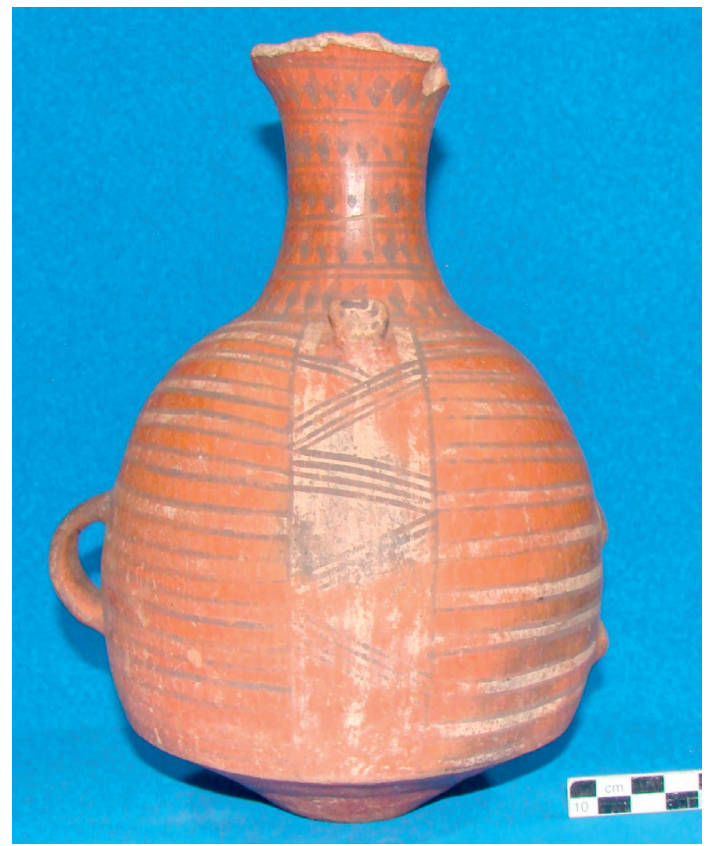

Figura 6. Aríbalo Inca Provincial (MEJBA 35100). Provincial Inca style aryballos (MEJBA 35100).

de que los administradores estatales lograran una estricta vigilancia de la producción (Earle 1994).

\section{Consideraciones Finales}

La cerámica inca, como emblema de poder y jerarquía, hallada en la kancha y en las viviendas que posiblemente estuvieron habitadas por los grupos vinculados a la política estatal podría estar reflejando la demarcación de estatus por parte de una minoría a cargo de la compleja organización socioeconómica del Pucará, y de la región como capital de Wamani. Esta cerámica presenta similitudes a la hallada en otras áreas nucleares del Tawantinsuyu, lo que remarca el prestigio de estas elites en el marco regional.

Por otro lado, la cerámica detectada en las casas-taller también sirve para reflexionar sobre el rol que tuvieron los artesanos especializados dentro de la sociedad. Vale mencionar que a diferencia de otras viviendas, en las que no se han detectado evidencias de trabajo productivo especializado o no presentan elementos que permitan caracterizarlas como edificios especiales, se han identificado piezas atribuibles a los estilos regionales incaicos y vasijas de fina calidad. Si bien estas piezas pudieron ser menos prestigiosas que la cerámica Inca Imperial o sus imitaciones, reflejan un trato preferencial, el que también se infiere a partir de la inclusión de otros bienes de destacado valor simbólico como keros (vasos de madera), tumis y discos de metal.

El trabajo lapidario y el metalúrgico quizás fue una de las clases de mita del área que más beneficios trajo al Estado. Estas labores también debieron ser reconocidas por los jefes locales, ya que mediante la entrega de sus producciones pudieron servir para el sostenimiento de alianzas, la redistribución y, principalmente, la reciprocidad en el tiempo. Otro aspecto que destaca los privilegios de estos artesanos es la presencia en los talleres de vasijas utilizadas para conservar y servir chicha, como cántaros, aribaloides y jarras, así como también de pucos para su consumo. Estas piezas podrían manifestar la tradición inca de proveer comida y bebida a los trabajadores corvée del Estado (Bray 2004).

Por último, un punto que resulta notorio en relación con la composición de los conjuntos analizados para las casas-taller es la baja proporción de vajilla Yavi-Chicha. De allí que no se pueda establecer si se contó con la presencia de mitmakunas chichas en el Pucará, a pesar que se sostenga que estos grupos se incorporaron a la población de la quebrada con las huestes incaicas (González 1982; Raffino 1993). No obstante, se debe considerar que para el desarrollo de la industria lapidaria se debieron trasladar artesanos desde otras provincias, ya que representó ser la imposición de una nueva tradición tecnológica y estilística nunca trabajada en la región (Otero 2013). La gran variedad de estilos cerámicos que componen los conjuntos del Pucará, además de la circulación de diferentes categorías de objetos, podría reflejar el movimiento de poblaciones destinadas a cumplir con esta y otras actividades productivas en la quebrada de Humahuaca.

Agradecimientos: A Gabriela Ammirati, Alejandra Reynoso, Victoria Coll, Silvia Manuale, Armando Mendoza y Presentación Aramayo, personal de los Depósitos de Arqueología de los Museos Etnográfico "J.B. Ambrosetti” y Arqueológico "Dr. E. Casanova" (FFyL-UBA) por su colaboración durante la revisión de la colección del Pucará de Tilcara. A los evaluadores por sus valiosos aportes, los que permitieron enriquecer este trabajo. Por último deseo agradecer a Myriam Tarragó, por su guía en el desarrollo de mis investigaciones, y a Beatriz Cremonte por sus acertados comentarios a partir de la lectura de este manuscrito. No obstante, los argumentos aquí vertidos son de mi responsabilidad. 


\section{Referencias Citadas}

Acuto, F.A. 2010. Living under the Imperial Thumb in the Northern Calchaquí Valley, Argentina. En Distant Provinces of the Inka Empire. Toward a Deeper Understanding of Inka Imperialism, editado por M. Malpass y S. Alconini, pp. 108-150. University of Iowa Press, Iowa City.

Bingham, H. 1913. In the Wonderland of Peru. The Work Accomplished by the Peruvian Expedition of 1912, under the Auspices of Yale University and the Nacional Geographic Society. Press of Judd \& Detweiler, Washington.

Bray, T.L. 2004. La alfarería imperial inka: una comparación entre la cerámica estatal del área de Cuzco y la cerámica de las provincias. Chungara Revista de Antropología Chilena 36:365-374.

Calderari, M. y V. Williams 1991. Re-evaluación de los estilos cerámicos incaicos en el Noroeste Argentino. En Imperio Inka, Actualización y Perspectivas por Registros Arqueológicos y Etnohistóricos, Vol. II. Revista Comechingonia, Año 9 - $\mathrm{N}^{\circ}$ especial, pp. 75-95. Córdoba.

Casanova, E. 1970. El Pucará de Tilcara (antecedentes, reconstrucción, guía). Publicación $\mathrm{n}^{\circ} 1$. FFyL. Museo del Pucará de Tilcara, Universidad de Buenos Aires.

Cook, A.G y M. Glowacki 2003. Pots, politics, and power: Huari ceramic assemblages and imperial administration. En The Archaeology and Politics of Food and Feasting in Early States and Empires, editado por T.L. Bray, pp. 173-202. Kluwer Academic/Plenum Publishers, New York.

Cremonte, M.B. 2006. El estudio de la cerámica en la reconstrucción de las historias locales. El Sur de la Quebrada de Humahuaca (Jujuy, Argentina) durante los Desarrollos Regionales e Incaico. Chungara Revista de Antropología Chilena 38:239-248.

Cremonte, M.B. 2014. El estilo Yavi-Chicha en instalaciones inkaicas del noroeste argentino. Las pastas como posible marcador identitario. En Ocupacion Inka y Dinámicas Regionales en los Andes (Siglos XV-XVII), editado por C. Rivera Casanovas, pp. 223-245. IFEA-Plural Editores, Serie Actas y Memorias IFEA, La Paz.

Cremonte, M.B. y L. Botto 2009. Unas vasijas especiales de contextos tardíos del Noroeste Argentino. Manufactura de los "Pucos Bruñidos". Estudios Atacameños 37:63-77.

D’Altroy, T.N., A.M. Lorandi y V.I. Williams 1994. Producción y uso de la cerámica en la economía política Inca. Arqueología 4:73-172.

D'Altroy, T.N., V.I. Williams y A.M. Lorandi 2007. The Inkas in the Southlands. En Variations in the Expression of Inka Power, editado por R. Burger, C. Morris y R. Matos M., pp. 85-133. Dumbarton Oaks, Washington DC.

Deambrosis, M.S. y M. De Lorenzi 1973. La influencia incaica en la Puna y Quebrada de Humahuaca, República Argentina. Revista del Instituto de Antropología 4:129-139.

Debenedetti, S. 1930. Las Ruinas del Pucará de Tilcara, Tilcara, Quebrada de Humahuaca (Pcia. De Jujuy). Archivos del Museo Etnográfico II, Primera Parte. Facultad de Filosofía y Letras, Universidad de Buenos Aires, Buenos Aires.

Earle, T. 1994. Wealth finance in the inka empire: evidences from the Calchaquí valley, Argentina. American Antiquity 59:443-450.
González, A.R. 1982. Las provincias inca del antiguo Tucumán. Revista del Museo Nacional XLVI:317-380.

Hyslop, J. 1992. Qhapaqñan. El Sistema Vial Incaico. Instituto Andino de Estudios Arqueológicos, Lima.

Julien, C.J. 2004. Las tumbas de Sacsahuaman y el estilo CuzcoInca. Ñawpa Pacha 25-27(1987-89):1-125.

Krapovickas, P. 1958/1959. Un taller de lapidario en el Pucará de Tilcara. RUNA 9:137-151.

López, M.A. 2004. Tecnología Cerámica en La Huerta, Quebrada de Humahuaca, Provincia de Jujuy, República de Argentina. Tesis para optar al grado de Doctor en Arqueología. Facultad de Filosofía y Letras, Universidad de Buenos Aires, Buenos Aires.

Meyers, A. 1975. Algunos problemas en la clasificación del estilo incaico. Pumapunku 8:7-25.

Morris, C. 1995. Symbols to power: Styles and media in the Inka State. En Style, Society and Person, Archaeological and Ethnological Perspective, editado por C. Carr y J.E. Neitzel, pp. 419-433. Plenum Press, New York.

Nielsen, A. 2001. Evolución social en la Quebrada de Humahuaca. En Historia Argentina Prehispánica, Tomo I, editado por E.E. Berberián y A.E. Nielsen, pp. 171-264. Editorial Brujas, Córdoba.

Otero, C. 2006. Análisis Cerámico del Recinto 2 de la Unidad 1, Sector Corrales del Asentamiento Urbanizado de Tilcara (SJuj Til 1-UH 1). Tesis para optar al título de Licenciado en Ciencias Antropológicas, orientación Arqueología. Facultad de Filosofía y Letras, Universidad de Buenos Aires, Buenos Aires.

Otero, C. 2013. Producción, Uso y Circulación de Bienes en el Pucará de Tilcara (Quebrada de Humahuaca, Jujuy). Tesis para optar al título de Doctor de la Universidad de Buenos Aires en Arqueología. Facultad de Filosofía y Letras, Universidad de Buenos Aires, Buenos Aires.

Otero, C. y M. Cremonte 2014. Local vessels technology of the Pucará of Tilcara during Inca Period (Quebrada of Humahuaca, Argentina). Journal of Anthropological Archaeology 33:108-118.

Otero, C. y P.A. Ochoa 2011. Primeras aproximaciones a la materialización del tiempo y las prácticas productivas especializadas en Tilcara (Quebrada de Humahuaca, Jujuy). Estudios Sociales del NOA. Nueva Serie 11:101-122.

Otero, C. y M.C. Rivolta 2013. Nuevas interpretaciones para la secuencia de ocupación de Tilcara (Quebrada de Humahuaca, Jujuy). Intersecciones en Antropología, en prensa.

Palma, J.R. 1998. Curacas y Señores: una Visión de la Sociedad Política Prehispánica en la Quebrada de Humahuaca. Editorial de la Facultad de Filosofía y Letras, Universidad de Buenos Aires, Buenos Aires.

Pardo, L. 1939. Clasificación de la cerámica cuzqueña. Revista del Museo e Instituto Arqueológico 4 (6-7):3-27.

Raffino, R.A. 1993. Inka. Arqueología, Historia y Urbanismo del Altiplano Andino. Ediciones Corregidor, Buenos Aires.

Runcio, M.A. 2009. Estilos e Identidades: Producción y Consumo de Vasijas Cerámicas en la Quebrada de Humahuaca durante los Períodos Tardío e Inca (900-1536 d.C.). Tesis para optar al grado de Doctor en Arqueología. Facultad de Filosofía y Letras, Universidad de Buenos Aires, Buenos Aires. 
Rowe, J.H. 1944. An Introduction to the Archaeology of Cuzco. Papers of the Peabody Museum of American Archaeology and Ethnology, vol. XXVII, $n^{\circ} 2$.

Rowe, J.H. 1946. Inca Culture at the Time of the spanish conquest. En Handbook of South American Indians, Vol. 5, editado por J. Steward, pp. 183-330. Washington DC.

Santoro, C., V.I. Williams, D. Valenzuela, A. Romero y V.G. Standen 2010. An archaeological perspective on the inka provincial administration of the South-Central Andes. En Distant Provinces of the Inka Empire. Toward a Deeper Understanding of Inka Imperialism, editado por M. Malpass y S. Alconini, pp. 44-74. University of Iowa Press, Iowa City.

Shimada, I. (ed.) 2007. Craft Production in Complex Societies. Multicraft and Producer Perspectives. The University of Utah Press, Utah.

González, L.R. y M.N. Tarragó 2004. Dominación, resistencia y tecnología: la ocupación incaica en el Noroeste argentino. Chungara Revista de Antropología Chilena 36:303-406.

Valcárcel, L.R. 1934a. Sajsawaman redescubierto. Revista del Museo Nacional 3(1-2):3-36. Lima.

Valcárcel, L.R. 1934b. II. Primer informe sobre los trabajos arqueológicos que se verifican en el Departamento de Cuzco. Revista del Museo Nacional 3(1-2):180-191. Lima.
Valcárcel, L.R. 1934c. Los trabajos arqueológicos del Cusco. II.- Sajsawaman redescubierto. Revista del Museo Nacional 3(3):211-233. Lima.

Valcárcel, L.R. 1935a. Los trabajos arqueológicos en el Dpto. del Cusco. Sajsawaman redescubierto (III). Revista del Museo Nacional 4(1):1-24. Lima.

Valcárcel, L.R. 1935b. Los trabajos arqueológicos en el Dpto. del Cusco. Sajsawaman redescubierto (IV). Revista del Museo Nacional 4(2):163-204. Lima.

Williams, V.I. 2004. Poder estatal y cultura material en el Kollasuyu. Boletín de Arqueología Pontificia Universidad Católica del Perú 8:209-245.

Williams, V.I., C.M. Santoro, A.L. Romero G., J. Gordillo, D. Valenzuela y V.G. Standen 2009. Mecanismos de Dominación Inka en los Valles Occidentales y Noroeste Argentino. Andes (Boletín del Centro de Estudios Precolombinos de la Universidad de Varsovia) 7:615-624.

Zaburlín, M.A. 2009. Historia de ocupación del Pucará de Tilcara (Jujuy, Argentina). Intersecciones en Antropología 10:89-103.

Zaburlín, M.A. y C. Otero 2013. Un manuscrito olvidado de J.B. Ambrosetti: "Exploraciones arqueológicas en la antigua ciudad del Pukará de Tilcara". Colección Saberes. Editorial de la Facultad de Filosofía y Letras, Universidad de Buenos Aires, en prensa.

\section{Notas}

1 La alfarería de estilo Pacajes, con una larga tradición en el Altiplano boliviano, durante la expansión incaica incorporó atributos de la cerámica cuzqueña (Williams 2004). Se trata de uno de los estilos que para esta época circularon ampliamente por el Kollasuyu. De allí que en este análisis se lo incluya dentro del grupo cerámico Inca Provincial no local. Si bien hasta el momento no se pueden determinar las variaciones de las pastas o las procedencias de los ejemplares aquí analizados, debido a que se trata de piezas a los que no se pudo tomar muestras para hacer estudios petrográficos de las pastas, ni FRX por el método aplicado, por presentar una buena conservación, vale mencionar que se cuenta con el análisis de la pasta de un fragmento de un plato ornitomorfo recolectado de superficie por la Dra. Cremonte en el Pucará de Tilcara. La pasta de esta pieza presenta similitudes con las pastas de la cerámica Yavi-Chicha (ver Cremonte et al. 2014 en este mismo número).

2 MEJBA: refiere a la numeración de la pieza según el catálogo del Museo Etnográfico "J. B. Ambrosetti". MT: numeración según el registro del Museo Arqueológico "Dr. E. Casanova” del Instituto Interdisciplinario Tilcara. 